\title{
On Investigating the Cognitive Complexity of Designing the Group Decision Process
}

\author{
Constantin-Bala Zamfirescu ${ }^{1}$, Luminita Duta ${ }^{2}$, Barna Iantovics ${ }^{3}$ \\ ${ }^{1}$ Lucian Blaga University of Sibiu, Faculty of Engineering, \\ Department of Computer Science and Automatic Control, \\ Emil Cioran 17, Sibiu, Romania, \\ zbc@acm.org \\ ${ }^{2}$ University Valahia of Targoviste, Faculty of Electrical Engineering, \\ Department of Computer Science and Automation, \\ Unirii Ave., 18-20, Târgovişte, Romania, \\ duta@valahia.ro \\ ${ }^{3}$ Petru Maior University of Targu Mures, Faculty of Sciences and Letters, \\ Department of Mathematics and Informatics, \\ Nicolae Iorga 1, Targu Mures, Romania, \\ ibarna@science.upm.ro
}

Abstract: The paper investigates the cognitive complexity associated with the design of group decision processes (GDP) in relation with some basic contextual factors such us task complexity, users' creativity and problem space complexity. The analysis is done by conducting a socio-simulation experiment for an envisioned software tool that acts as collaborative environment for the GDP design. The simulation results provide some insights on how to engineer context-adaptable functionalities aiming at minimizing the cognitive complexity associated with the GDP design. Although the research is carried out for a specific case, namely the GDSS technology, the results may be easily replicated for any sort of collaborative working environment where the cognitive complexity associated with its effective use is playing an important role.

Keywords: group decision support systems, facilitation, social simulation.

\section{Introduction}

Group Decision Support System (GDSS) is an interactive computer-based environment that supports concerted and coordinated team effort towards completion of joint tasks [1]. This collaborative environment is made up from a collection of highly configurable tools (i.e., brainstorming, voting and ranking, multi-criteria analysis, action planning, agenda setting etc.) which require a high level of expertise for an effective use to support complex decisions [2]. The strong relationship between the GDP outcome and the presence of a skilful facilitator to properly configure the available tools is thoroughly presented in many field studies of GDSS research [3]. Nevertheless, the presence of a scarce resource, such as a skilful facilitator, rapidly becomes the most demanding challenge in the wide spread of GDSS technology in organizations. To reduce the dependence on the facilitator, the participantdriven GDSS was proposed as a promising direction to leverage the skills and abilities of each group member [4]. However, this approach is highly constrained by the cognitive complexity associated with the construction, coordination and execution of GDP by inexperienced users.

To overcome the problem of cognitive complexity Briggs and de Vreede [5] have introduced the thinkLet (TL) concept, defined as a discrete decomposition unit that integrates a specific software tool, its configuration and a script specifying the proper usage of the tool. Consequently a TL may be considered a predefined interaction protocol among the GDSS's users, an interaction mediated and structured by a tool from the GDSS software package. As a result, the GDP is structured as a flow of discrete interactions, each of them being reflected in the specific TL that codifies the essential knowledge to execute collaborative processes.

The paper investigates the complexity associated with the GDP design in relation with some basic contextual factors such us the problem complexity, the users' creativity and the problem space complexity. The remaining part of the paper is organized as it follows. The next section describes the main components of an envisioned collaborative software tool that act as a collaborative working environment for the GDP design. These components are implemented and 
tested in a socio-simulation experiment described in Section 3. As in many field studies of GDSS research, the experimental results show clear self-organizing capabilities as regards the longitudinal use of the collaborative environment to design the GDP, but simultaneously a high dependability of performance on the contextual factor. From the engineering standpoint of constructing purposeful facilitation tools for e-meetings, these results are discussed and concluded in the last section.

\section{The Simulation Model the GDP design}

The socio-simulation model is developed from the perspective of envisioning a collaborative software tool that is used by the GDSS's users to co-design the GDP. From this standpoint, the tool acts as a stigmergic environment that integrates and coordinates, on a social-scale, the facilitation knowledge of the GDSS's users. Conceptually this perspective is similar with the way in which, for instance, a collaborative CAD software is used to coordinates and integrates the specific engineering knowledge in the field architectural design [6]. Consequently, the socio-simulation basically mimics the users' conceptual 'navigation' over the semantic structure of the problem space composed of TLs. It implies the design of a population of agents and a shared environment where the agents are localized and moved over it. For the collaborative modelling of GDP the stigmergic environment is the software tool which support the manipulation of the conceptual problem space that comprise all the TLs discovered and documented by the users community (so far there are over 70 TLs acknowledged in literature [7]), while the agents are the users responsible to define, execute and evaluate the GDP (a path through the conceptual space of the available TLs).

\section{The semantic environment for the GDP design}

According to Parunak [8], a stigmergic environment assumes the definition of three main components: 1) the topology, 2) the states, and 3) the process. Structurally, the topology is usually represented as a fully connected weighted graph that codifies the facilitation knowledge of group decision (Figure 1). This knowledge presumes correlated information among the users and the TLs, reflecting the users' evaluation of the TL's performance (a node in the graph) relative to a problem type. The performances are stored for each "problem type" in a variable associated with each edge of the graph. The "problem type" is simply codified through a unique ID to distinguish among different performances when they are read, during the modelling phase of the GDP, or modified, after the GDP has been executed and evaluated by the agents. Evaluation of a GDP model entails a subjective assessment of the model, after its execution, against some performance criteria that quantifies the efficiency, effectiveness and satisfaction with the GDP. Note that for the rationale of our simulation the composite criteria to quantify the TL's performance are irrelevant, it just serves as a unified denominator to measure the GDP outcome.

The performances from all the graph's edges describe the state of the environment over time. Usually, the environment executes a set of processes on the variables (as aggregation end evaporation in the case of ants [8]). For the purpose of our simulation, we apply a simple additive rule to simulate the aggregation of performances. After the evaluation of a GDP model that corresponds to a certain problem type, a path through a number of $n$ nodes $T L_{1}, \ldots, T L_{n}$, the aggregation rule may takes the following form:

$P_{j, k}\left(T L_{k}, t\right)=P_{j, k}\left(T L_{k}, t-1\right)+P_{j, k}\left(T L_{k}\right) / \lambda$

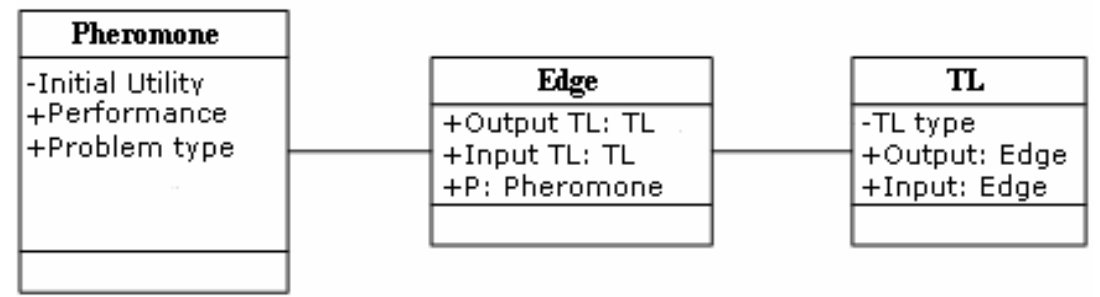

Figure 1. The simplified topology for the conceptual environment represented in UML. 
where $t$ represents the temporal component of the model which is incremented by one for each successive use of the GDSS - it practically corresponds to a simulation tick; $k$ is the TL's identification index from the set of TLs used to model the GDP; $P_{j, k}\left(T L_{k}\right)-$ is the performance of the $k$-th TL evaluated from the side of TL $j ; P_{j, k}\left(T L_{k}, t\right)$ and $P_{j, k}\left(T L_{k}, t-1\right)$ are the new and previous values of the performances stored on the edge between the TLs $j$ and $k$; and $\lambda$ is a tuning parameter, arbitrary chosen, to weight the impact of the last evaluation.

\section{The agents' behaviour over the semantic environment}

The agents are users who interact with the collaborative tool to design a GDP. Conceptually, at any time an agent is "located" in a node (TL) of the conceptual problem space, performing one of the following basic actions:

- evaluating the preference for the next possible TL (or TLs) that are going to be executed given the current context of the GDP implementation;

- selecting the next best TL (or a group of TLs) for further completing the GDP model;

- executing the TL (or the group of TLs) from the model, and finally;

- assessing the performance for the executed TLs.

The assessment activity is simulated using the formula (1), while the first three actions with Luce's selection axiom [9]:

$$
p_{j k}=e^{P_{j k}\left(T L_{k}\right) / T} / \sum_{i=1}^{m} e^{P_{j i}\left(T L_{i}\right) / T}
$$

where $p_{j k}$ represents the preference for an alternative TL, i.e. the selection probability of the TL $k$ from the TL $j ; i$ is the index of TLs connected from the side of node $j$ (in fact all the $m$ TLs available in the problem space as long the graph is fully connected); and $T$ is a positive subunitary parameter used to define the deviation from a pure rational behaviour (for $\mathrm{T}=1$ we have a random selection behaviour, while for $\mathrm{T}=0$ a deterministic one).

The above formula is the most common model of stochastic decisions due to its correlation with the psycho-social observations of human behaviour in several domains. As a result of normalization, the preferences for the unexploited TLs are diminishing after each performance update. This mechanism replicates the pheromone evaporation process of the real ants (e.g. even if a TL has been positively evaluated after an execution of a GDP model, the associated preference will decrease once a better alternative is discovered and more frequently used). Under complex circumstances when TL's selection depends on other users, or the performances available on the edges are uncertain or incomplete, or there is impossible to evaluate the performance of a TL due to any real constraint (i.e. temporal, cognitive, economic, etc.), we consider the user who models the GDP to have limited cognitive capacity (bounded rationality). Note that Luce's selection axiom does not specify the reasons for the "bounded rationality"; instead, it tries to generalize the selection behaviour of human decisionmakers through the parameter $\mathrm{T}$ which may be interpreted as the evaluation costs or uncertainty associated with the quantification of TLs' performance (on one side, when $\mathrm{T}=0$, there is no uncertainty associated with the TL selection, while on the other side, when $\mathrm{T}=1$, there is a completely random selection).

\section{Experimental Results}

To evaluate the cognitive complexity for modelling the GDP we conducted a virtual experiment implemented in the NetLogo multiagent simulation environment [10] (Figure 2). Note that the NetLogo implementation includes some additional variables which are beyond the scope of this paper.

The experiment presumes that users are facilitating the e-meeting by trying to codefine, from a metacognitive stance, the GDP model for a problem type. Defining the GDP model implies the conceptual navigation in the problem space in order to find the best sequence of TLs that maximise the model's performance. The model's performance is simply computed by averaging the "Initial Utility" values for the TLs that are composing the GDP. These values are randomly chosen and assigned when the simulation is initialized. 


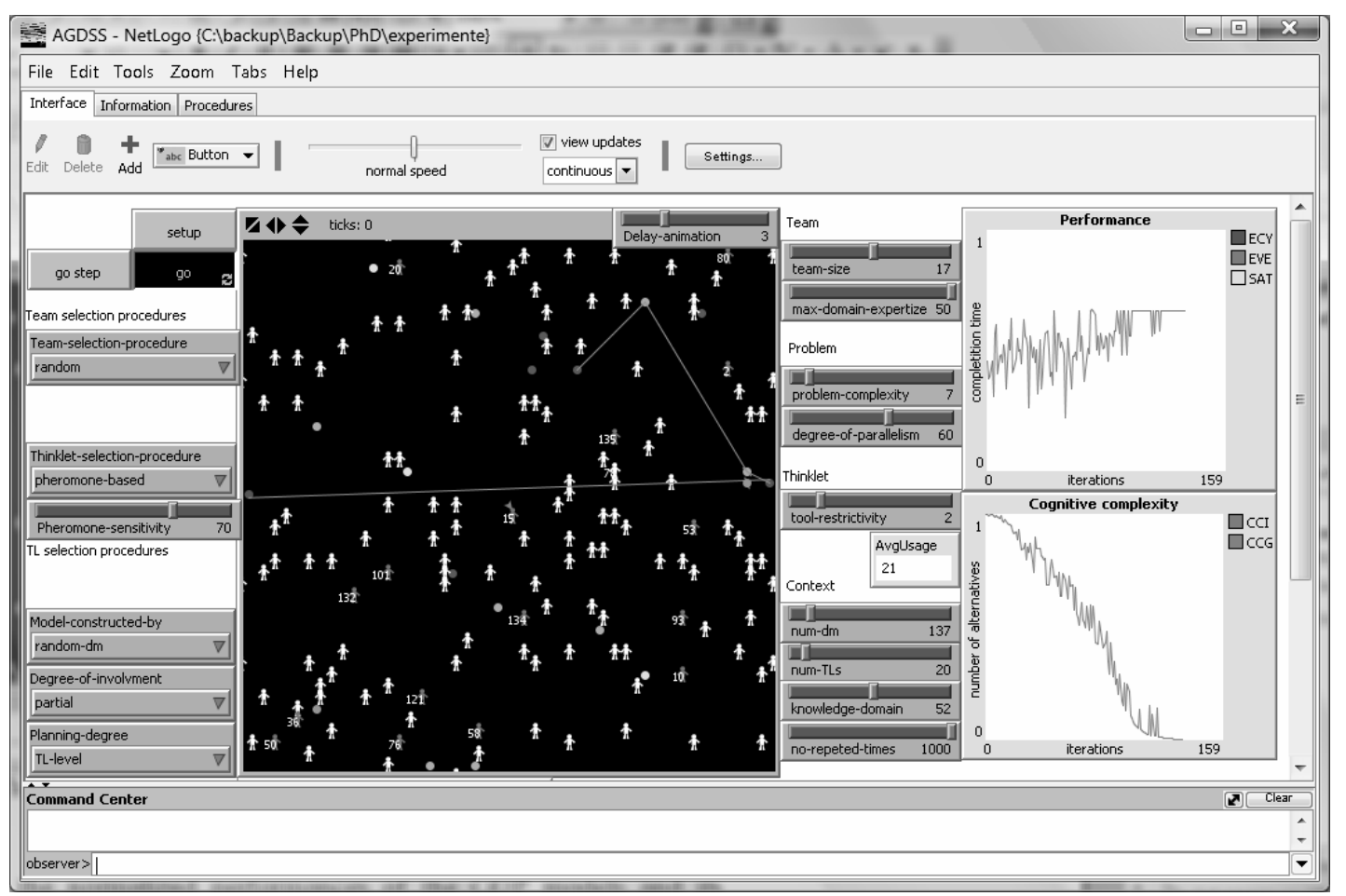

Figure 2. The interface of the NetLogo simulation environment for the implemented experiment.

The section presents the normalized entropy for 100 successive explorations (iterations) in relation with three factors that potentially could impact over the GDP models' performance:

- problem complexity $(\mathrm{PC})$ - defined as the number of distinct TLs that compose a GDP model;

- $\quad$ social temperature $(\mathrm{T})$ - which stands for the $\mathrm{T}$ parameter as defined in $\mathrm{Eq}(2)$; and

- complexity of the problem space (PS) defined as the total number of TLs that compose the GDP modelling problem space.

An exploration stands for a complete execution cycle of a GDP. It includes three consecutive phases: 1) finding a suitable model through the successive selection (using the $\mathrm{Eq}(2)$ ) of TLs that compose the GDP model for a given problem type; 2) executing the identified model and assessing its performance by reading and averaging the predefined performance values of all the TLs that compose the GDP model; 3) assessing the model by updating the performances values (using the $\mathrm{Eq}(1)$ ).

The statistics are aggregated from 30 experiments for a relatively simple set of experimental values for the observed parameters.
The auto-organization of relations between TLs (i.e. the performance update after successive evaluations) entails a decrease of freedom due to the emergence of contextual constraints that reduce, in time, the probability to select some TLs (i.e. the preference for the available TL as defined in $\mathrm{Eq}(2))$. For a problem type, the degree of freedom corresponds to the probabilistic distribution of preferences for the available alternatives that is equivalent with the Shannon normalized entropy [11][12]. The Shannon normalized entropy for the selection of a TL is given by:

$$
E\left(p_{j, k}\right)=-\sum_{k=1}^{m} p_{j, k} \ln \left(p_{j, k}\right) / \ln (m)
$$

where $p_{j k}$ - represents the preference, the selection probability of the TL $k$ from the TL $j ; i-$ is the index for the TLs connected from the node $j$ (in fact, all the $m$ TLs available in the problem space).

When the recorded performances are equal for all the available modelling alternatives, the user is considering the entire problem space when he selects a feasible TL (the probabilities from the $\mathrm{Eq}(2)$ being equally distributed entail an entropy equal with 1). Contrary, when the recorded performances 
favour a single alternative, the user will have no freedom in the selection of the best TL (all the probabilities from formula (2) being 0 excepting the best alternative that is 1 , entails an entropy equal with 0 ). Thus, the entropy associated with TL's selection is a measure of cognitive complexity for modelling the GDP. Moreover, it is a local metrics that can be computed for each TL's selection activity for modelling the GDP.

\section{The impact of problem complexity experimental variable over the cognitive complexity of GDP design}

Figure 3 shows the cognitive complexity associated with the GDP modelling for different values of the $\mathrm{PC}$ experimental variable. The data is obtained for a problem space composed of $30 \mathrm{TLs}$ and $\mathrm{T}=0.7$. Since this measure is computed on the basis organizational settings where the complex problems are often less frequent and consequently there is not an adequate amount of opportunities to explore the GDSS functionalities. On the other hand, problem complexity concerns the users' satisfaction in the GDP design as well. PC is recognized by many authors to often be a subjective factor that measures the availability of relevant information [13]. The more informed/ predictable the GDP design is (i.e. the individual entropy smaller) the smaller is the subjective users' perception over the problem complexity. Consequently, the cognitive complexity for complex problems may be lessening by incorporating functionalities that provide relevant information that minimize the problem space for each discrete activity of GDP modelling. As for any design tool, these basic activities are the selection and elaboration of a model.

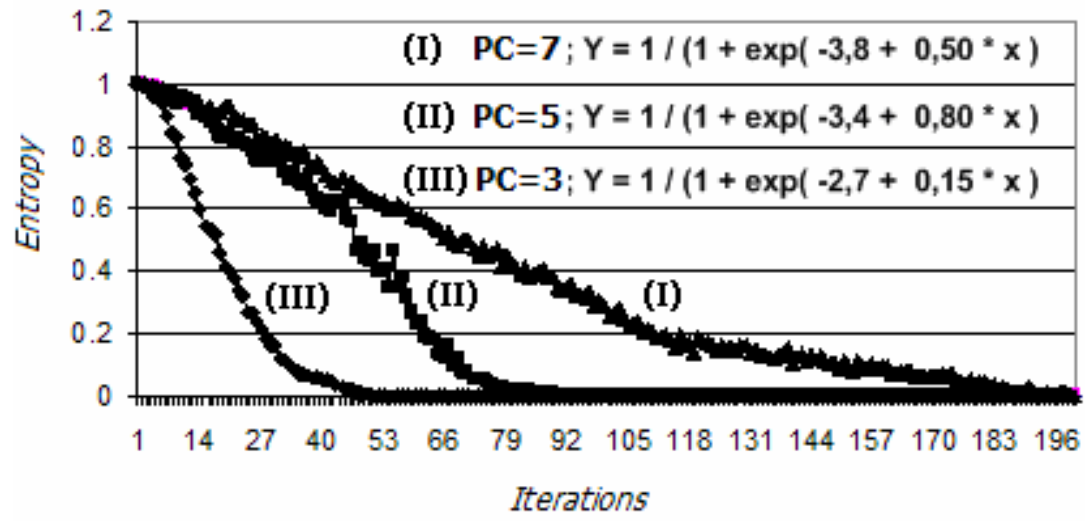

Figure 3. The normalized entropy of the GDP design for different problem complexities.

of the local data for each selection action (the performances available on the outward edges from the current TL), the figure corresponds to the average of entropies for all the TL selection actions needed to complete the GDP model $(3,5$ and 7 successive TLs, depending on the value assigned to the PC experimental variable).

The data from the Figure 3 shows that problem complexity has a great impact over the entropy (around 190 iterations are required to decrease the entropy close to 0 for a $\mathrm{PC}=7$, while less than 5 iterations are needed for a $\mathrm{PC}=3$ ). These results prove that for complex problems there is an increasing need for experimentation, learning and creative use of the GDSS. At the same time, they contrast with the real use of GDSS in

\section{The impact of social temperature experimental variable over the cognitive complexity of GDP design}

Figure 4 shows the cognitive complexity associated with the GDP design for different values of $T$. The data is obtained for a problem space and a PC composed of 30 and 5 TLs respectively. The performances are better for higher values of $\mathrm{T}$ as a result of exhaustive exploration of the problem space. Consequently, when the design problem for a GDP is in the learning phase, it is preferably to encourage a creative use of the GDSS by neglecting the suggestions offered as a result of computing the collective preferences. Obviously, this issue presumes a high frequency of that problem 


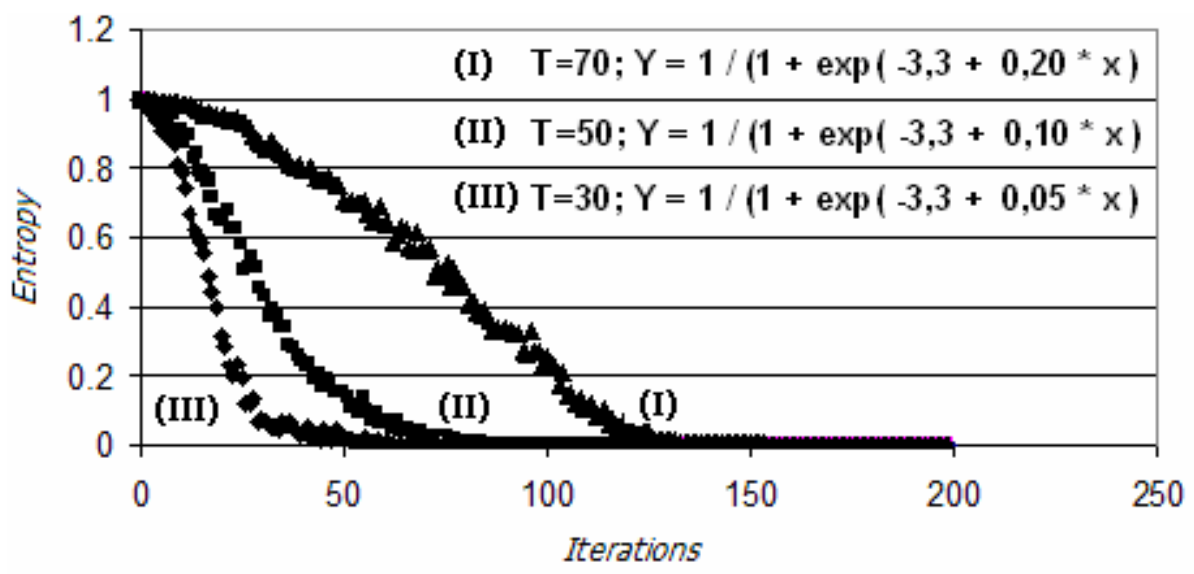

Figure 4. The normalized entropy of the GDP design for different T values.

type and a long-term use of the GDSS in organization to compensate the extra time required for experimentation purpose as depicted in Figure 5. Note that as long the $\mathrm{T}$ parameter measures the degree in which preferences are considered by the users during the GDP design, at the same time it may be used as a post factum measure to quantify the users' creativity.
The impact of problem space complexity experimental variable over the cognitive complexity of GDP design

Figure 6 shows the cognitive complexity associated with the GDP modelling in a problem space with a different number of TLs. The data is obtained for a simple problem type composed of three TLs with $\mathrm{T}=0.7$. One can

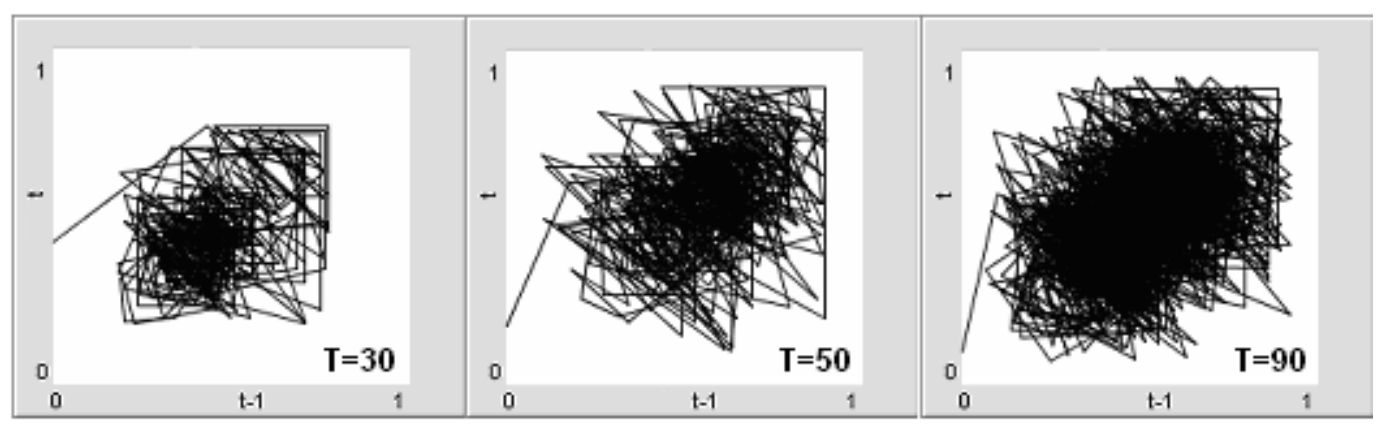

Figure 5. The transition maps of the GDP performance for different $\mathrm{T}$ values.

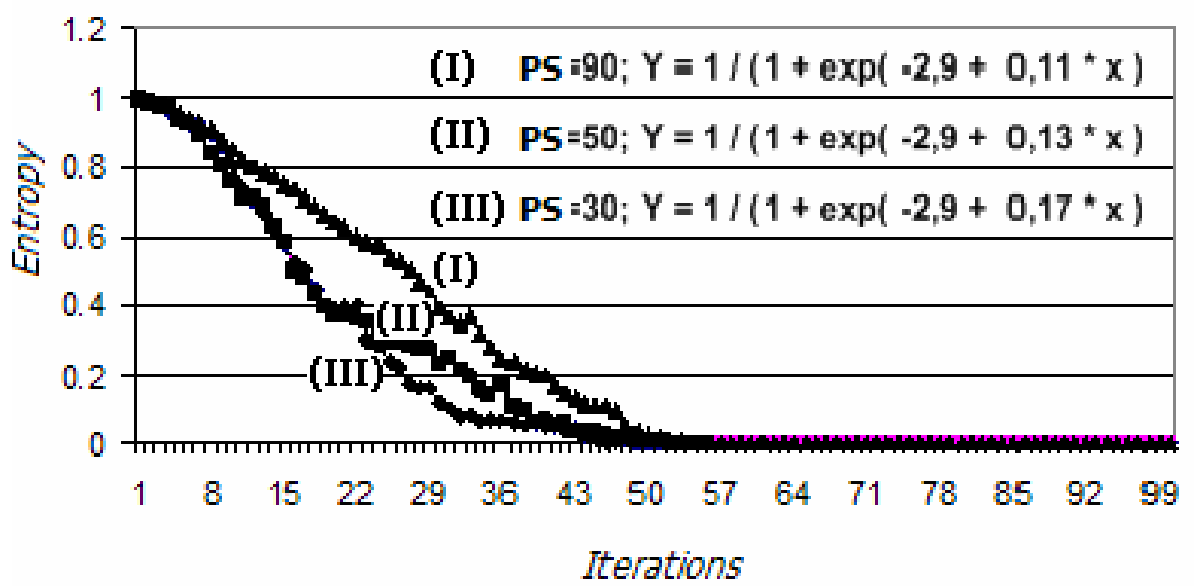

Figure 6. The normalized entropy for the GDP design in a problem space of different dimensions. 
notice that the complexity of the problem space has basically no impact over the convergence of the entropy function. This is one of the core arguments for employing stigmergic coordination mechanisms for global optimization problems which remains scalable and effective in open, dynamic and uncertain environments. On the other hand, an increase of available TLs for modelling the GDP will automatically result in an increase of alternatives to model it. In the GDSS research field has been experimentally shown that, as the number of decision alternatives are growing, the decision makers are tempted to consider less of them [14]. This implies an accelerated discrimination of the possible alternatives through the intensification of the GDP model evaluation.

\section{Conclusions}

The work from this paper investigated some of the basic contextual factors (such us the problem complexity, the users' creativity and the problem space complexity) that have a significant impact over the cognitive complexity associated with GDP design in emeetings. The investigation has been conducted by implementing and testing in a sociosimulation experiment an envisioned collaborative software tool that acts as a stigmergic environment for modelling the GDP.

The results extend the conclusions presented in [15][16], showing that the dominant factor for the wide adoption of GDSS technology in real organizations still remains the problem complexity. It may be lessening by incorporating functionalities that provide relevant information for the GDP design (i.e. the knowledge resulted from the subjective evaluation of each GDP from a large community of users) that entails a greater need for experimentation, learning and creative use of the GDSS. Moreover, the performances are better for higher values of the social temperature as a result of exhaustive exploration of the problem space. Consequently it is preferably to encourage a creative use of the GDSS when the GDP modelling problem is in the learning phase. Conversely, the complexity of the problem space has basically no impact over the cognitive complexity associated with the GDP design. This shows why the emergent functionalities of a facilitation tool for the GDP design should be engineered around some simple stigmergic coordination mechanisms.

\section{REFERENCES}

1. DESANCTIS, G., B. GALlUPE, A Foundation for the Study of Group Decision Support Systems, Management Science, 1987, pp. 589-609.

2. FILIP F. G., Decision Support and Control for Large-scale Complex Systems, Annual Reviews in Control, vol. 32(1), 2008, pp. 61-70.

3. NIEDERMAN F., C. M. BEISE, P. M. BERANEK, Issues and Concerns about Computer-Supported Meetings: The Facilitator's Perspective, MIS Quarterly, vol. 20(1), 1996, pp. 1-22.

4. HELQUIST J. H., J. KRUSE, M. ADKINS, Developing Large Scale Participant-Driven Group Support Systems: An approach to Facilitating Large Groups, Proceedings of the First HICSS Symposium on Field and Case Studies of Collaboration, IEEE Computer Society Press, Los Alamitos, CA, 2006.

5. BRIGGS R. O., G. J. DE VREEDE, J. F. NUNAMAKER JR., Collaboration Engineering with ThinkLets to Pursue Sustained Success with Group Support Systems, Journal of Management Information Systems, vol. 19(4), 2003, pp. 31-63.

6. CHRISTENSEN L. R., The Logic of Practices of Stigmergy:

Representational Artifacts in Architectural Design, Proceedings of the 2008 ACM Conference on Computer Supported Cooperative Work, ACM, New York, NY, 2008, pp. 559-568.

7. DE VREEDE G. J., R. O. BRIGGS, G. L. KOLFSCHOTEN, ThinkLets: A Pattern Language for Facilitated and Practitioner-Guided Collaboration Processes, International Journal of Computer Applications in Technology, vol. 25, 2006, pp. 140-154.

8. PARUNAK. H. V. D., A Survey of Environments and Mechanisms for Human-Human Stigmergy, Lecture 
Notes on Artificial Intelligence, vol. 3830, Springer, 2006, pp. 163-186.

9. LUCE D., Individual Choice Behaviour, Wesley, New York, 1959.

10. WILENSKY U., NetLogo, Center for Connected Learning and Computer-Based Modeling, Northwestern University, http://ccl.northwestern.edu/netlogo/, 1999.

11. GUERIN S., D. KUNKLE D., Emergence of Constraint in SelfOrganizing Systems. Nonlinear Dynamics, Psychology, and Life Sciences, vol. 8(2), 2004, pp. 131-146.

12. PARUNAK V. H. D., S. BRUECKNER, Entropy and Self-Organization in Multi-Agent Systems. Proceedings of the Fifth International Conference on Autonomous Agents, Montreal, Canada, 2001, pp. 124-130.
13. BYSTRÖM, K., K. JÄRVELIN, Task Complexity Affects Information Seeking and Use, Information Processing \& Management, vol. 31, 1995, pp. 191-213.

14. POOLE M. S., J. ROTH, Decision Development in Small Groups IV: A Typology of Group Decision Paths, Human Communication Research, vol. 15(3), 1989, pp. 323-356.

15. C.B. ZAMFIRESCU, An AgentOriented Approach for Supporting Self-Facilitation in Group Decisions, Studies in Informatics and Control, vol. 12, 2003, pp. 137-148.

16. ZAMFIRESCU, C.B., F.G. FILIP, Swarming Models for Facilitating Collaborative Decisions, International Journal of Computers, Communication and Control, vol. V, 2010, http://journal.univagora.ro/download/pdf/ 397.pdf, pp. 125-137. 